\title{
Biomarkers of Cardiovascular Responses among War Veterans with Posttraumatic Stress Disorder
}

\author{
Borzoo Amirpour ${ }^{1 *}$, Alireza Aghayousefi ${ }^{1}$, Rahim Goli $^{2}$, Leila Abdolmaleki ${ }^{1}$ \\ ${ }^{1}$ Department of Psychology, Payam-e-Noor University, Tehran, Iran
}

${ }^{2}$ Unit martyr Foundation and Veterans Affairs of Kermanshah, University of Applied Science and Technology, Kermanshah, Iran

\section{ABSTRACT}

Introduction: Due to the possible impact of posttraumatic stress disorder (PTSD) on biological markers of cardiovascular responses, it has become an essential part and hallmark of the diagnostic and statistical manual of mental disorders in the Fifth edition-DSM-5. This study aimed to investigate the biomarkers of cardiovascular responses in war veterans with PTSD. Materials and Methods: 34 male war veterans, among veterans with posttraumatic stress disorder in Kermanshah, participated in this cross sectional study on 2014. They were approved by medical committee as patients who suffer from PTSD. The data were collected by PTSD Checklist-Military version (PCL-M). Results: Data indicate that the mean age, the percentage of injury, and the duration that each individual spent in the war were 53.73, 45.72, and 4.07, respectively. The mean scores of systolic blood pressure, diastolic blood pressure, heart rate, and body temperature were $123.72 \mathrm{~mm} \mathrm{Hg}, 82.94 \mathrm{~mm} \mathrm{Hg}, 89$, and $36.51^{\circ} \mathrm{C}$, respectively. In addition, the Pearson correlation coefficient among the biomarkers of cardiovascular and the total score obtained in the posttraumatic stress disorder checklist was positive and significant. Conclusion: The results indicate the biological markers of cardiovascular responses as a risk factor for cardiovascular diseases in the war veterans with PTSD.

* Corresponding Author: Borzoo Amirpour

E-mail: borzooamirpour@gmail.com 


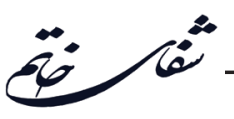

\title{
نشانگر هاى زيستى پاسخهاى قلبى -عروقى در ميان جانبازان جنَ مبتلا به اختلال استرس يس از سانحه
}

\author{
برزو امير يور'”. عليرضا آقايوسفى'، رحيم كَلى'، ليلا عبدالملكى' \\ اكروه روانشناسى، دانشَاه بيام نور، تهران، ايران \\ זواحد بنياد شهيد و امور ايثاركران كرمانشاه، دانشكاه علمى كاربردى، كرمانشاه، ايران
}

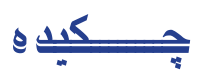

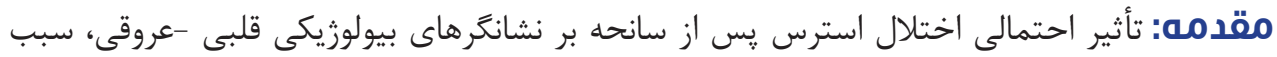

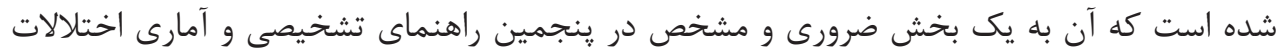

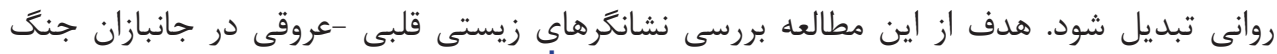

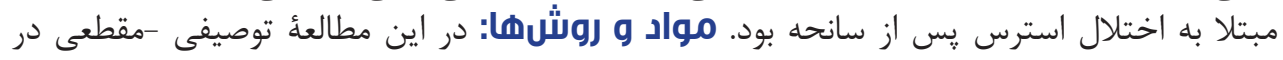

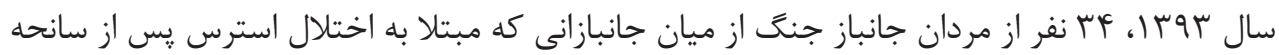

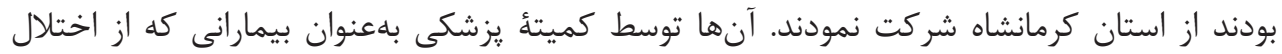

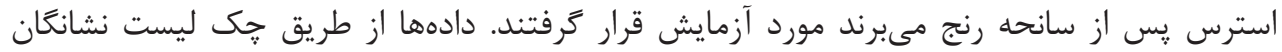

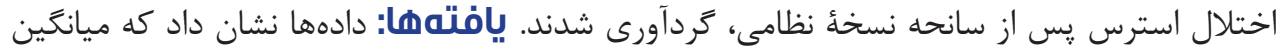

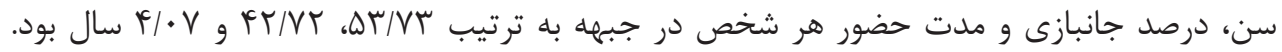

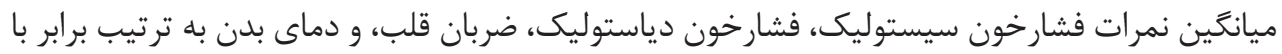

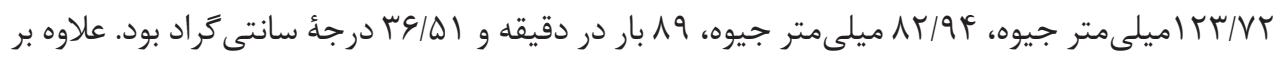

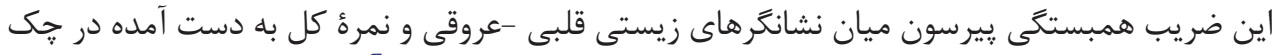

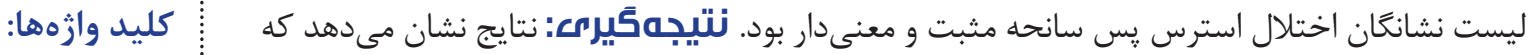

1. إنستنشانكر ها r r إنبازان r. r. اختلال استرس يس از سانحه

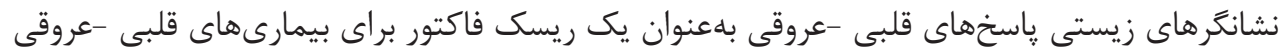

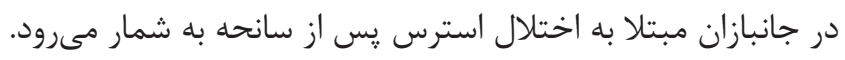


قلبى -عروقى آن بهعنوان ييامد قرار گرفتن در معرض استرس

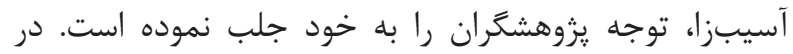

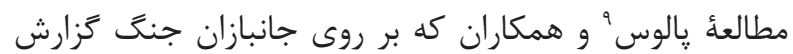

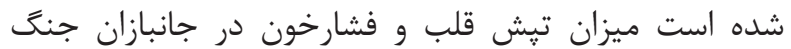

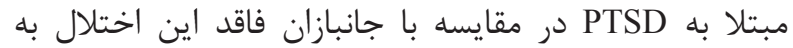

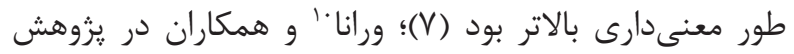

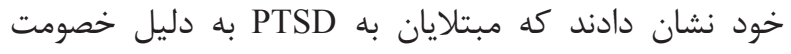

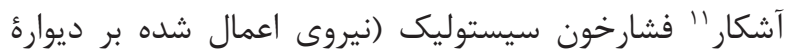

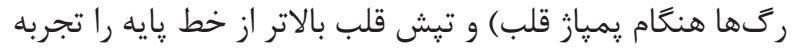

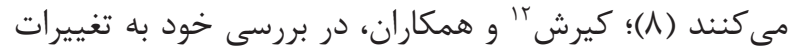

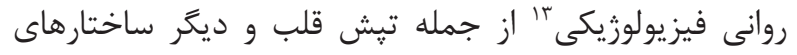

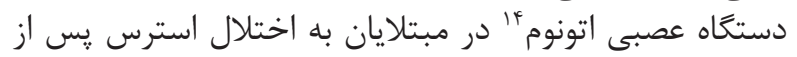
سانحه اذعان داشتهاند (9).

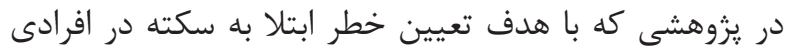

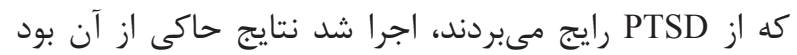

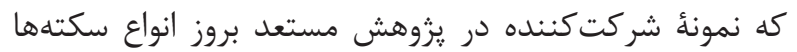

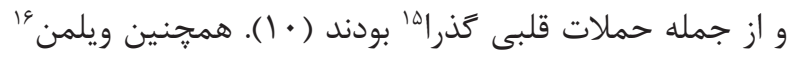

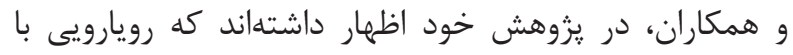

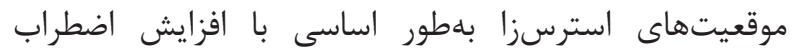

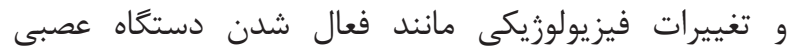

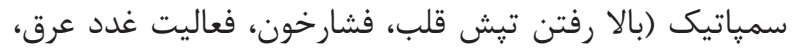

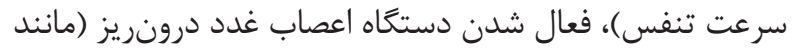

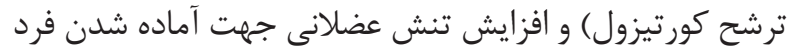

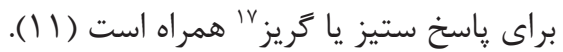

نكتئ مهمم در ارتباط با مبتلايان به PTSD، فعال بودن دستخاه

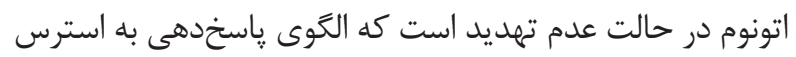

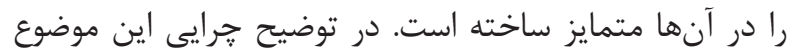

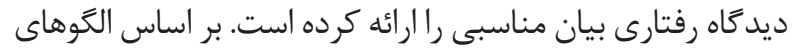

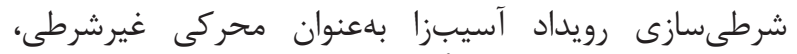

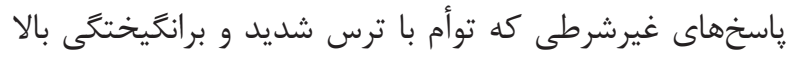

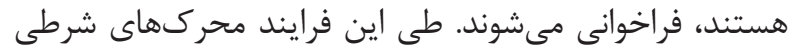

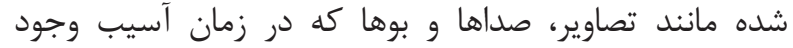

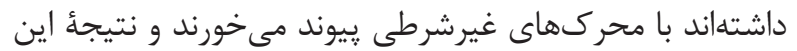

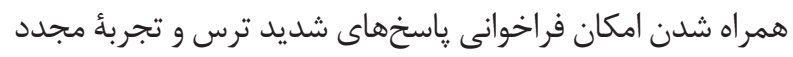

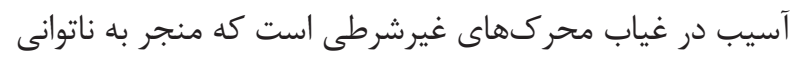

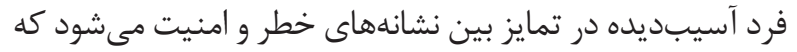

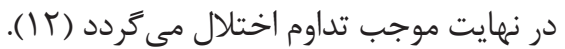

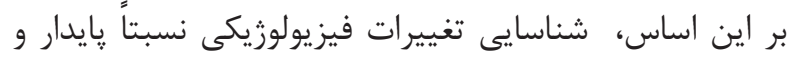

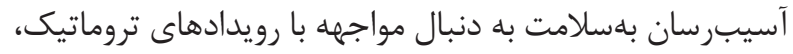

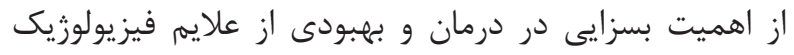

${ }^{1}$ Posttraumatic stress disorder (PTSD)

${ }^{2}$ Trauma-and stressor- related disorders

${ }^{3}$ Helplessness or horror

${ }^{4}$ Socio-occupational impairments

${ }^{5}$ Epidemiology

${ }^{6}$ Cardiovascular biomarkers

${ }^{7}$ Biomarkers

${ }^{8}$ World Health Organization (WHO)

${ }^{9}$ Paulus
ويزگ اساسى همأ موجودات زنده توانايى واكنش، مقابله و

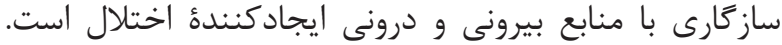

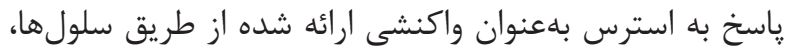

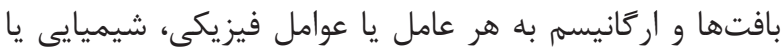

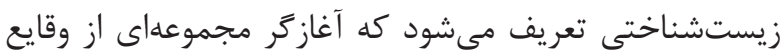

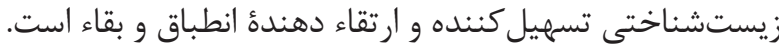

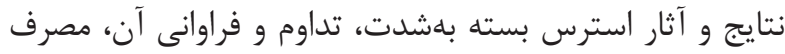

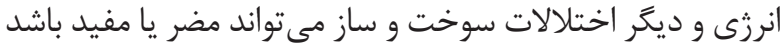

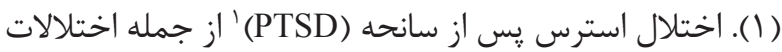

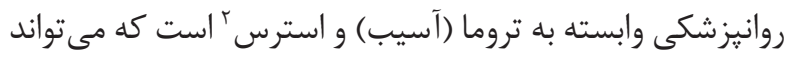

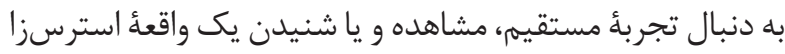

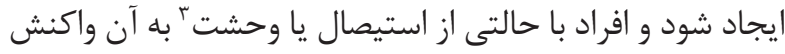

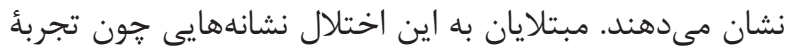

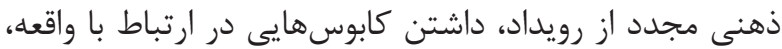

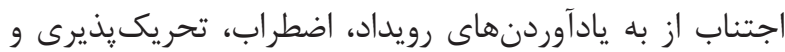

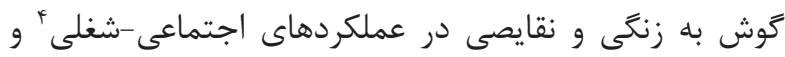
زيستشناختى را نشان مى نقهند (r)

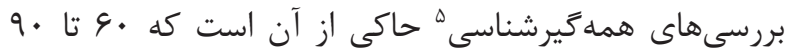

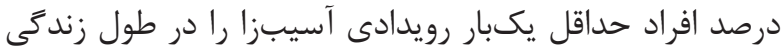

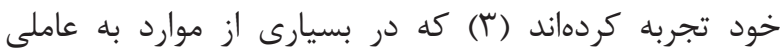

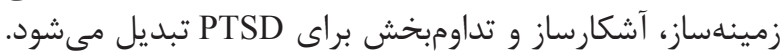

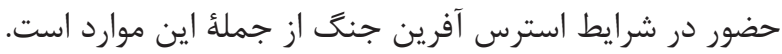

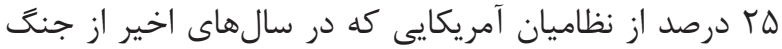

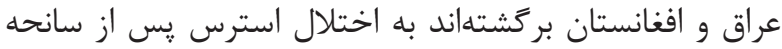

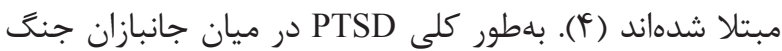

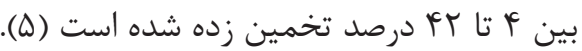

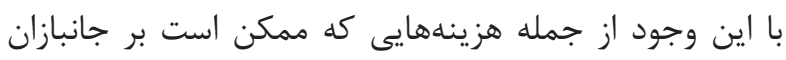

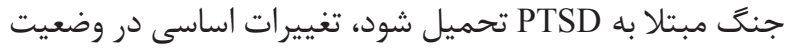

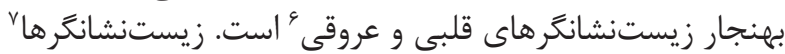

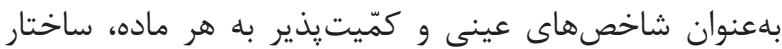

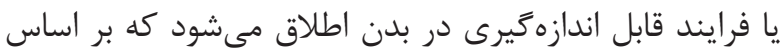

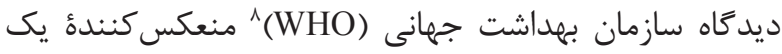

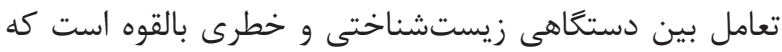

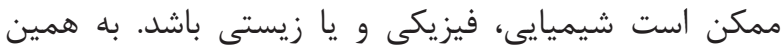

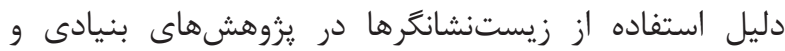

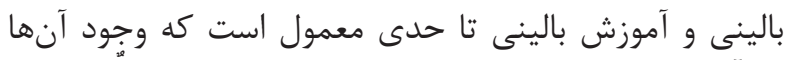

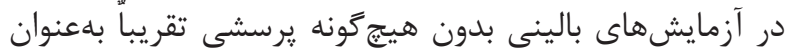

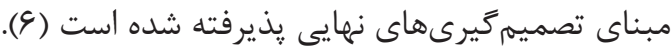

توجه به تغييرات ايجاد شده در زيستنشانكرها از جمله نوع
${ }^{10}$ Vrana
${ }^{11}$ Covert hostility
${ }^{12}$ Kirsch
${ }^{13}$ Psychophysiological alterations
${ }^{14}$ Autonomic nervous system
${ }^{15}$ Ischemic
${ }^{16}$ Willmann
${ }^{17}$ Fight-or-flight response 
شامل: وجود اختلال سايكوتيك و دوقطبى فعال، وابستگى إنى

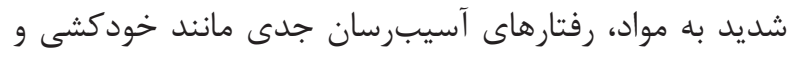

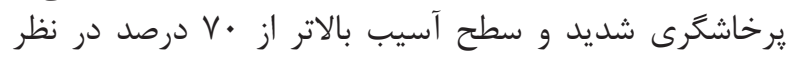

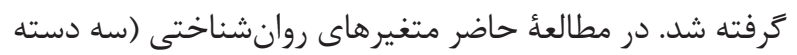

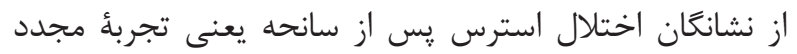

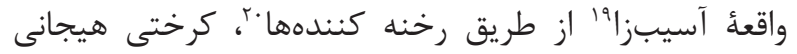

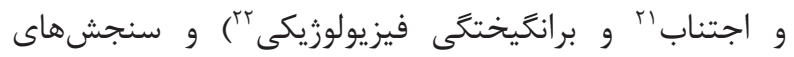

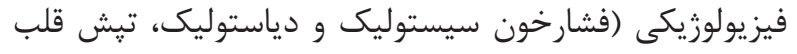

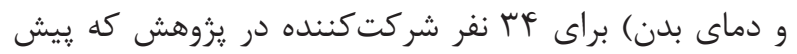

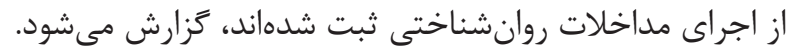

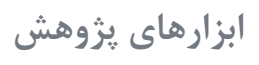

جى ليست اختلال استرس يس از سانحه -نسخهُ نظامى

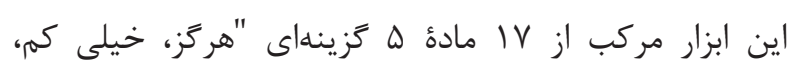

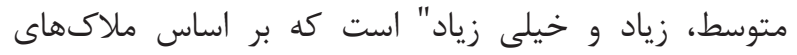

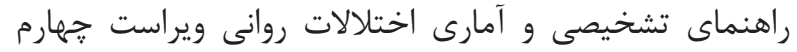

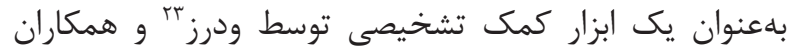

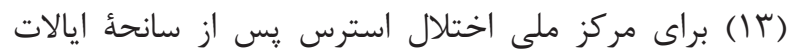

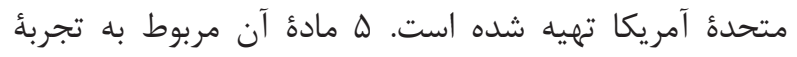

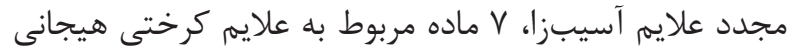

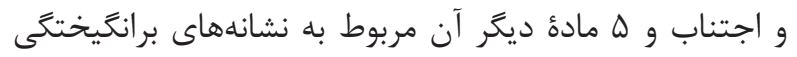

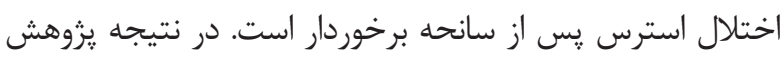

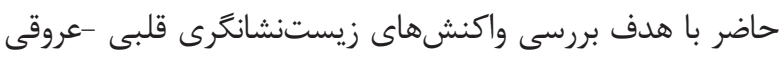

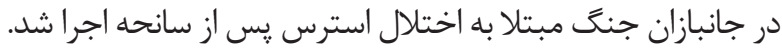

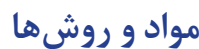

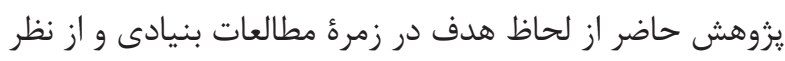

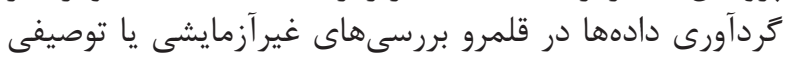

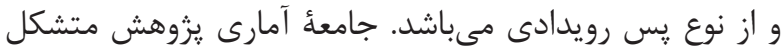

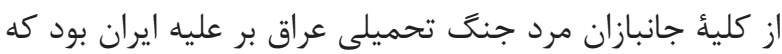

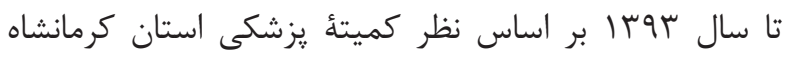

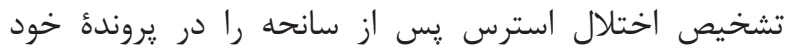

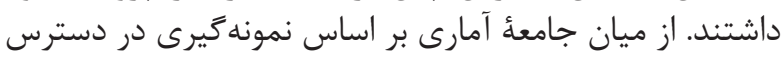

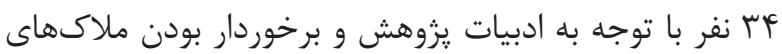

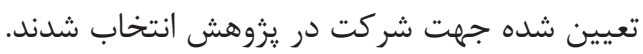

ملاكهاى ورود به يزوهش جانبازان مرد، دارا بودن مواردى إنى

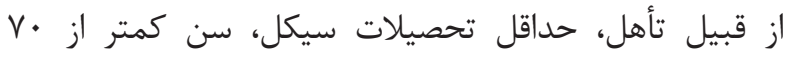

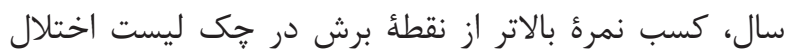

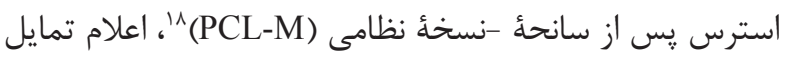

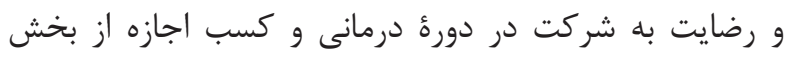

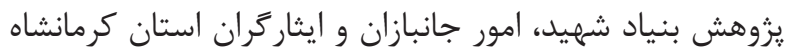

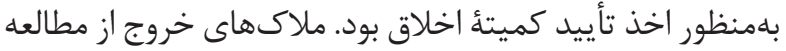

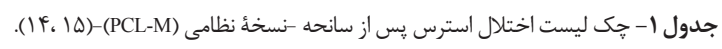

\begin{tabular}{|c|c|}
\hline تويهها & رديف \\
\hline تكرار خاطرات، افكار يا تصاوير ناراحتكنيده از يك تجربه استرسآور جنكى & 1 \\
\hline رؤياهاى تكرارى ناراحتكنينده در خواب و بيدارى از تجربئ استرسآور جنكى & T \\
\hline 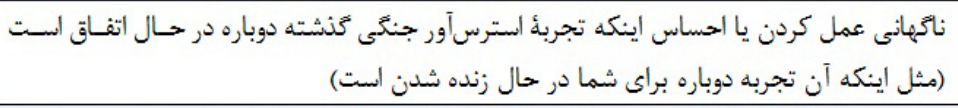 & r \\
\hline 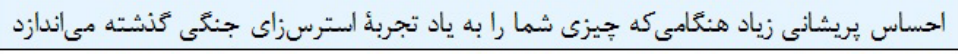 & F \\
\hline 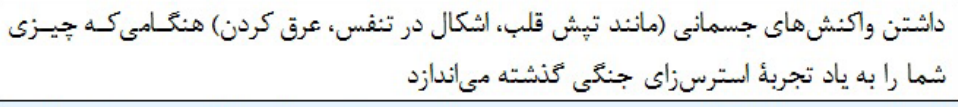 & $\Delta$ \\
\hline داشتن اجستاب و دورى از تفكر ياصـات دربارة آن & 9 \\
\hline 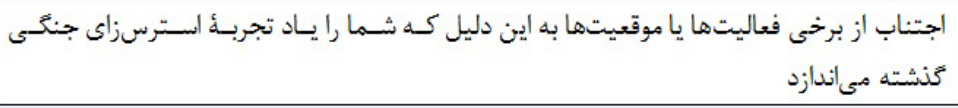 & $v$ \\
\hline زحمت و سختى در به يادآوردن قسمتهاى مههم يكى تجربهُ جنكّى استرسزا & $\wedge$ \\
\hline از دست دادن علاقه و لذت، از فعاليتهاى كه قبلاً از آنها لذت مىبرديد & 9 \\
\hline احساس فاصله يا بريدگى از ديكرًان & 1. \\
\hline 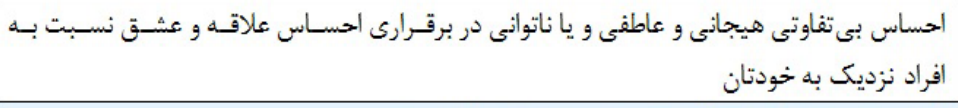 & 11 \\
\hline داثتن اين احساس كه آيندة شما به طريقى كوتاه شده است & $\pi$ \\
\hline اشكال در به خواب رفتن و تداوم خواب & ir \\
\hline احساس تحريكيذيرى يا داشتن حملات خشم & If \\
\hline مشكل براى داشتن تمركز بر كارها و امور زندكى & 10 \\
\hline فوقالعاده هوشيار بودن يا مراقب بودن (كوش بدزنكى) يا در حالت دفاعى بودن & 19 \\
\hline احساس از جا يريدن و يا بدآسانى از جا يريدن & iv \\
\hline
\end{tabular}

${ }^{18}$ The post-traumatic stress disorder checklist-military (PCL-M)

${ }^{19} \mathrm{Re}$-experiencing of the traumatic event

${ }^{20}$ Intrusion
${ }^{21}$ Emotional numbing and avoidance

${ }^{22}$ Physiological arousal

${ }^{23}$ Weathers 


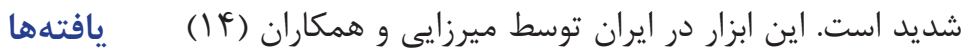

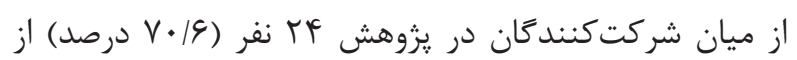
جانبازان فيزيكى و •ا نفر (

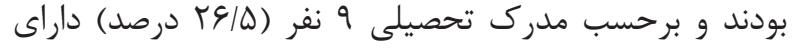

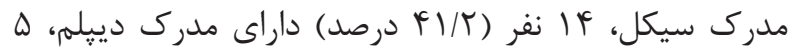

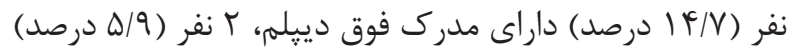

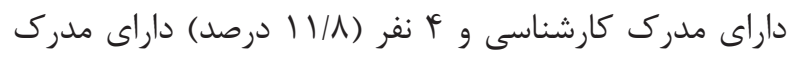

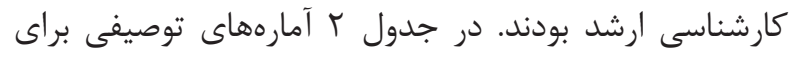

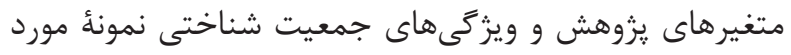

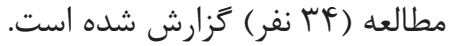

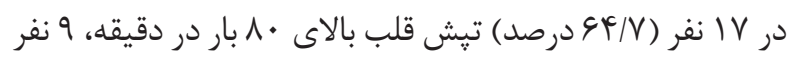

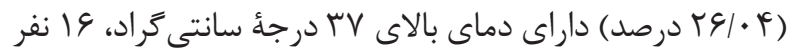

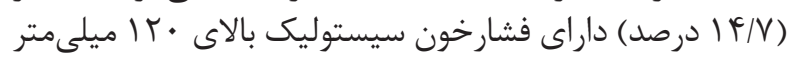

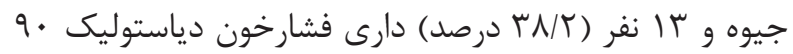

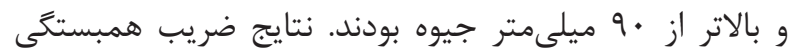

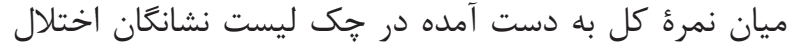

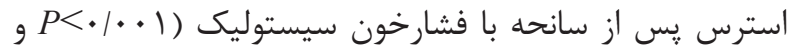

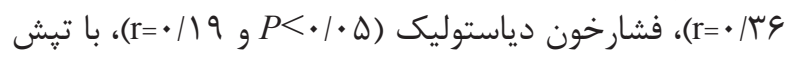

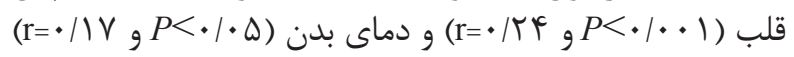

$$
\text { مثبت و معنى دار بود. }
$$

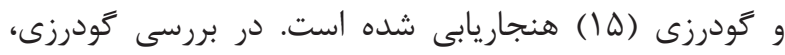

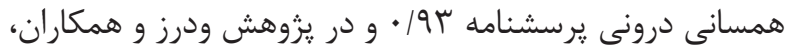

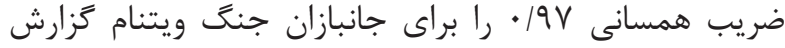

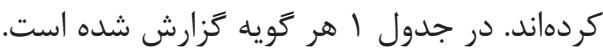
فشارسنج و دماسنج رقمى شركت آلمانى بيورر

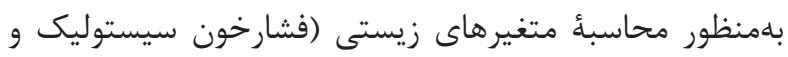

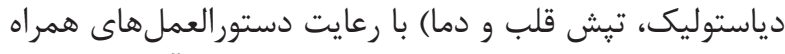

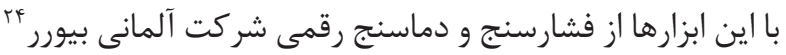

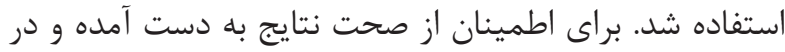

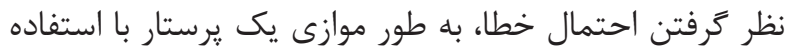

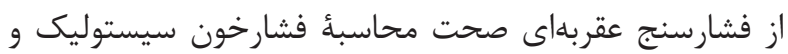
دياستوليك را تأييد مى فنمود.

$$
\text { تجزيه و تحليل آمارى دياستوليد }
$$

دادهها بلهورت درصد، فراوانى و ميانكين \pm انحراف معيار

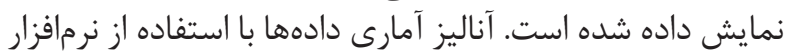

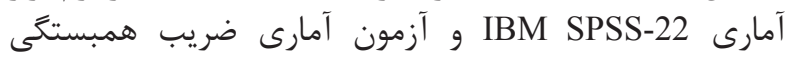

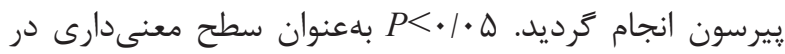

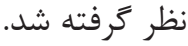

\begin{tabular}{|c|c|c|c|c|}
\hline 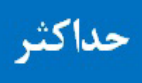 & 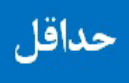 & انحراف معيار & 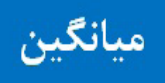 & متغير ها \\
\hline GF & id & $\Delta / \Delta r$ & $\Delta r$ & سن (سال) \\
\hline v & 1 & $1 / 49$ & $\mathrm{~F}_{\mathrm{N}} / \cdot \mathrm{V}$ & مدت حضور در جنع (ماه) \\
\hline$\vee \cdot$ & r. & $11 / 09$ & FT/NT & درصد جانبازى \\
\hline r & Ir & $r / 9 \Lambda$ & $1 N / \Delta \theta$ & تجربهُ مجدد رويداد آسيبزا \\
\hline r & 11 & $r / \cdot r$ & TG/DT & اجتناب و كرختى هيجانى \\
\hline Tr & IV & $1 / 1 r$ & $r \cdot N q$ & برانكيختكى فيزيولوزيكى \\
\hline VT & $\Delta \varphi$ & $r / \Delta \Delta$ & $90 / 11$ & 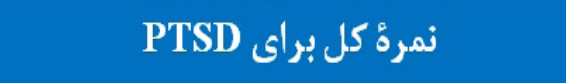 \\
\hline If. & 11. & $\Lambda / \Delta \cdot$ & ITHNT & فشارخون سيستوليك (ميلىمتر جيوه) \\
\hline$q$. & $V$. & $9 / \pi 9$ & $\Lambda r / q r$ & فشارخون دياستوليك (ميلى متر جيوه) \\
\hline 94 & VD & R/Tr & $\wedge 9 / \cdots$ & تيش قلب (تيش در دقيقه) \\
\hline$r V / 4$. & $r</ q$. & $\cdot / 0 \Delta 9$ & $r \varphi|\Delta|$ & لماى بدن (درجهُ سانتى كراد) \\
\hline
\end{tabular}

جدول r- شاخصهاى توصيفى كسب شده در متغيرها و ويزگى هاى جمعيت شناختى (n=rF).

${ }^{24}$ Beurer's digital barometer and thermometer 


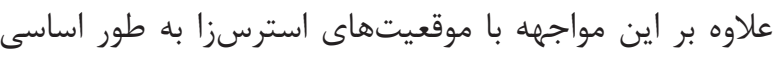

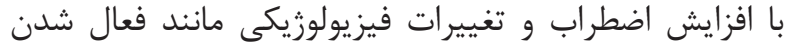

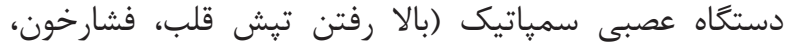

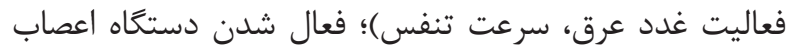

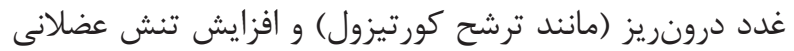

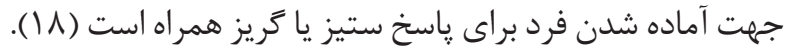

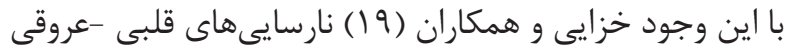

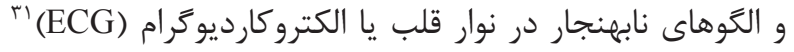

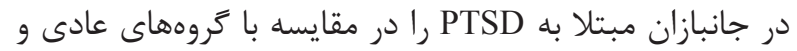

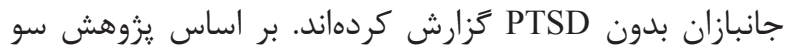

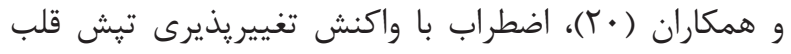

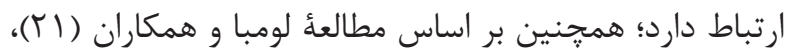

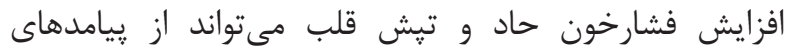
اضطراب و استرس مزمن باشد.

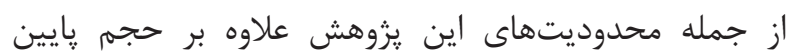

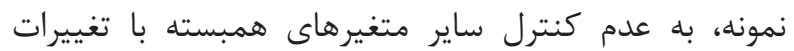

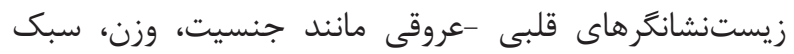

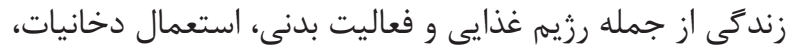

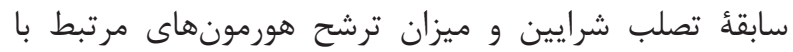

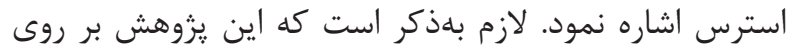

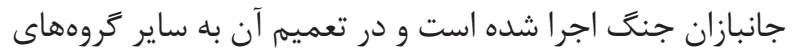

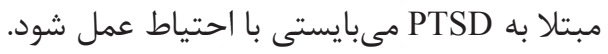

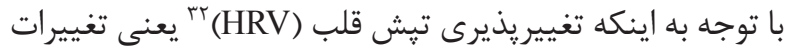

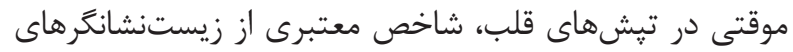

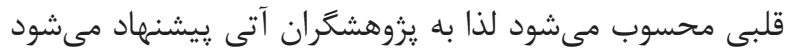

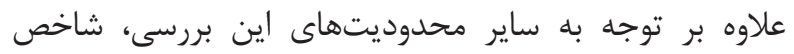

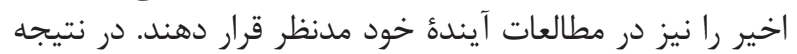

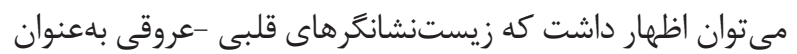

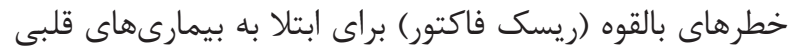

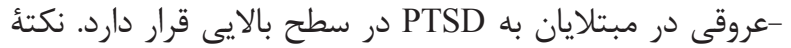

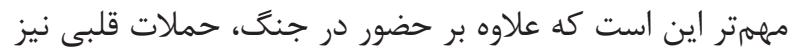

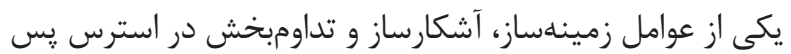

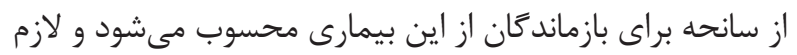

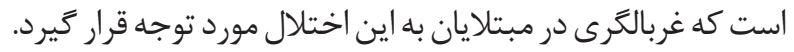

$$
\text { تشكر و قدردانى }
$$

مقالهٔ حاضر از رسالهُ دكتراى آقاى برزو اميريور اقتباس كرديده

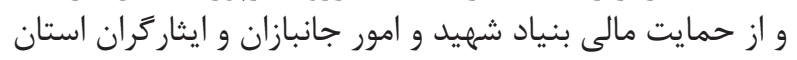

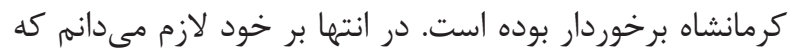

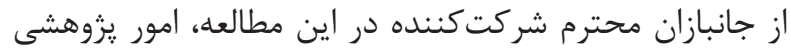

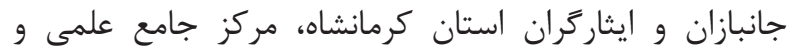

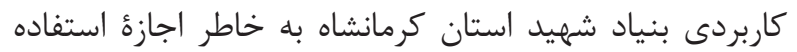

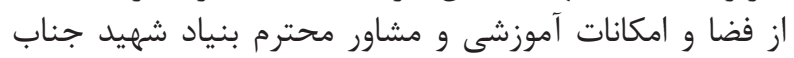

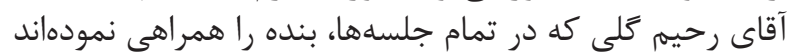

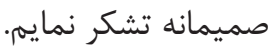

\footnotetext{
${ }^{25}$ Hypothalamic-pituitary-adrenal axis (HPA)

${ }^{26}$ Sympathetic adrenomedullary system

${ }^{27}$ A disease biomarker

${ }^{28}$ Pathogenic processes
}

\section{بحث و نتيجهَ}

بيشرفتهاى قابل توجهى در رابطه با درك مبانى زيستشناختى إنى

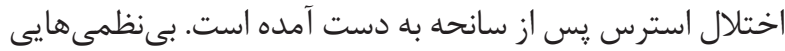

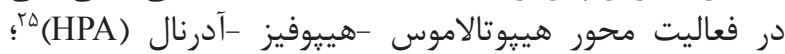

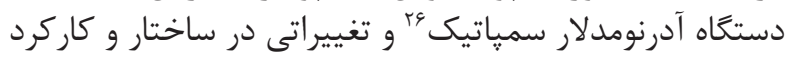

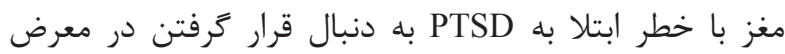

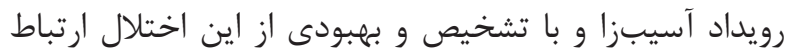

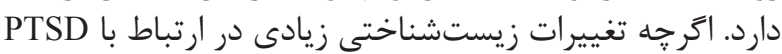

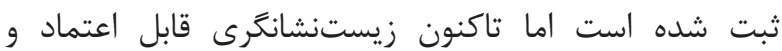
اختصاصى براى اين اختلال تعيين نشده است.

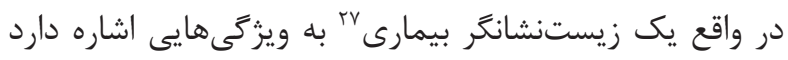

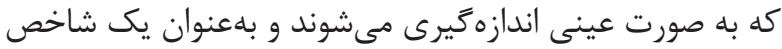

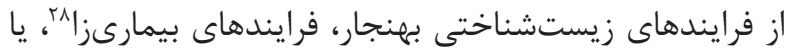

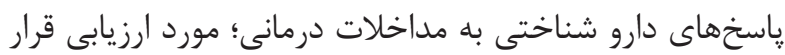

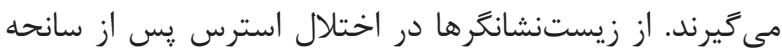

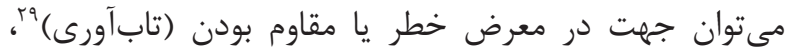

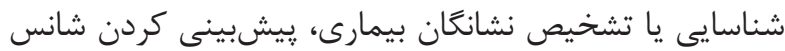

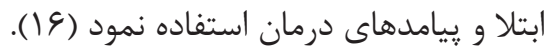

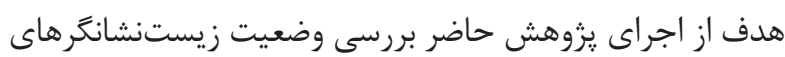

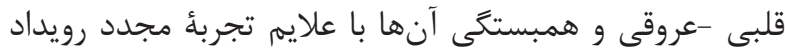

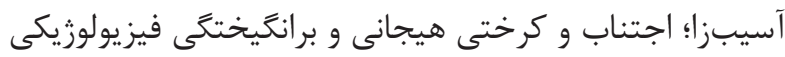

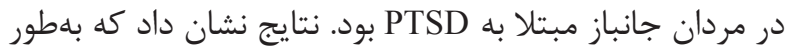

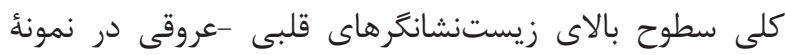

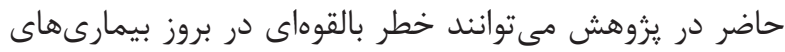

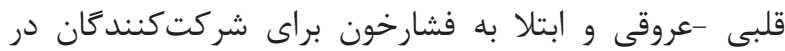

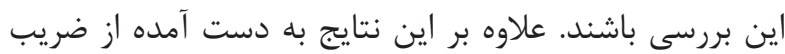

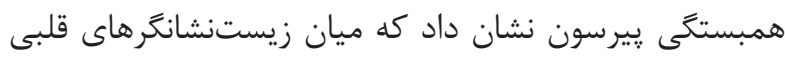

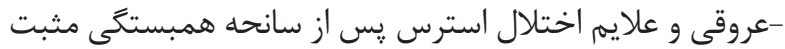

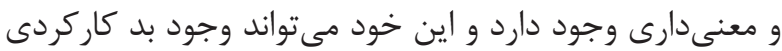

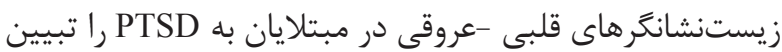
و حتى ييشبينى كند.

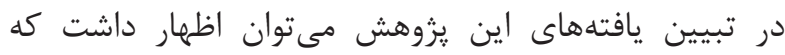

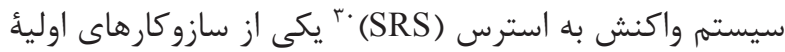

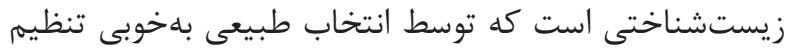

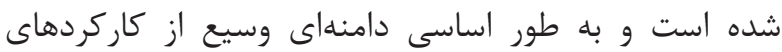

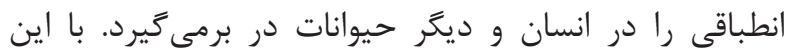

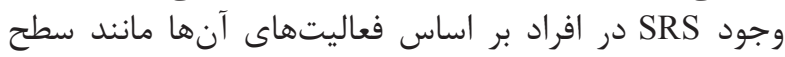

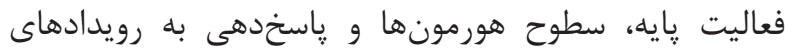

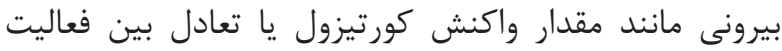

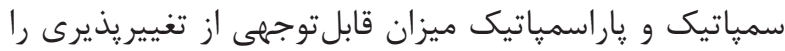

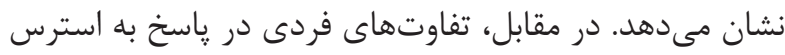

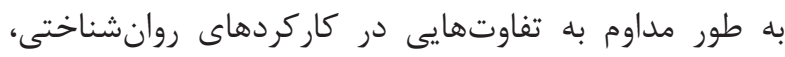
روابط اجتماعى و خطريذيرى ابتلا به اختهارئ اختلالات روانى ورانى جسمانى ارتباط دارد (IV) (IV)

\footnotetext{
${ }^{29}$ Risk/resilience

${ }^{30}$ Stress response system

${ }^{31}$ Electrocardiogram

${ }^{32}$ Heart rate variability (HRV)
} 
1. Demirovic D, Rattan SI. Establishing cellular stress response profiles as biomarkers of homeodynamics, health and hormesis. Exp Gerontol. 2013; 48(1): 94-8.

2. Abbey G, Thompson SB, Hickish T, Heathcote D. A meta-analysis of prevalence rates and moderating factors for cancer-related post-traumatic stress disorder. Psychooncology. 2014; 24(4): 371-81.

3. Morris MC, Rao U. Psychobiology of PTSD in the acute aftermath of trauma: Integrating research on coping, HPA function and sympathetic nervous system activity. Asian J Psychiatr. 2013; 6(1): 3-21.

4. Meyers LL, Strom TQ, Leskela J, Thuras P, KehleForbes SM, Curry KT. Service utilization following participation in cognitive processing therapy or prolonged exposure therapy for post-traumatic stress disorder. Mil Med. 2013; 178(1): 95-9.

5. Cesur R, Sabia JJ, Tekin E. The psychological costs of war: military combat and mental health. J Health Econ. 2013; 32(1): 51-65.

6. Aghayousefi A, Amirpour B, Alipour A, Zare H. Effect of cognitive processing therapy on cardiovascular biomarkers of veterans with post-traumatic stress disorder. IJWPH. 2015; 7(1): 43-8.

7. Paulus EJ, Argo TR, Egge JA. The impact of posttraumatic stress disorder on blood pressure and heart rate in a veteran population. J Trauma Stress. 2013; 26(1): 169-72.

8. Vrana SR, Hughes JW, Dennis MF, Calhoun PS, Beckham JC. Effects of posttraumatic stress disorder status and covert hostility on cardiovascular responses to relived anger in women with and without PTSD. Biol Psychol. 2009; 82(3): 274-80.

9. Kirsch V, Wilhelm FH, Goldbeck L. Psychophysiological characteristics of pediatric posttraumatic stress disorder during script-driven traumatic imagery. Eur J Psychotraumatol. 2015; 6: 25471. doi: 10.3402/ejpt.v6.25471.

10. Chen MH, Pan TL, Li CT, Lin WC, Chen YS, Lee $\mathrm{YC}$, et al. Risk of stroke among patients with posttraumatic stress disorder: nationwide longitudinal study. Br J Psychiatry. 2015; 206(3): 302-7.

11. Willmann M, Langlet C, Hainaut JP, Bolmont B. The time course of autonomic parameters and muscle tension during recovery following a moderate cognitive stressor: dependency on trait anxiety level. Int J Psychophysiol. 2012; 84(1): 51-8.

12. Sijbrandij M, Engelhard IM, Lommen MJ, Leer A, Baas JM. Impaired fear inhibition learning predicts the persistence of symptoms of posttraumatic stress disorder(PTSD). J Psychiatr Res. 2013; 47(12): 1991-7.

13. Weathers FW, Litz BT, Herman DS, Huska JA, Keane TM. The PTSD Checklist (PCL): Reliability, validity and diagnostic utility. San Antonio; 9th Annual Meeting of the International Society for Traumatic Stress Hisser Studies. 1993.

14. Mirzaee J. Karami GR, Ameli J, Hemmati MA. Investigation of clinical diagnosis by psychological tests in PTSD outpatients and inpatients. J Mil Med. 2004; 6 (3): 201-8.

15. Goodarzi MA. Evaluating validity and reliability of Mississippi post-traumatic stress disorder scale. J psychol. 2003; 7(2): 153-78.

16. Lehrner A, Yehuda R. Biomarkers of PTSD: military applications and considerations. Eur J Psychotraumatol. 2014; 14; 5. doi: 10.3402/ejpt.v5.23797.

17. Del Giudice M, Ellis BJ, Shirtcliff EA. The adaptive calibration model of stress responsivity. Neurosci Biobehav Rev. 2011; 35(7): 1562-92.

18. Willmann M, Langlet C, Hainaut JP, Bolmont B. The time course of autonomic parameters and muscle tension during recovery following a moderate cognitive stressor: dependency on trait anxiety level. Int J Psychophysiol. 2012; 84(1): 51-8.

19. Khazaie H, Saidi MR, Sepehry AA, Knight DC, Ahmadi M, Najafi F, et al. Abnormal ECG patterns in chronic post-war PTSD patients: a pilot study. Int J Behav Med. 2013; 20(1): 1-6.

20. Suh S, Ellis RJ, Sollers JJ 3rd, Yang HC, Emery $\mathrm{CF}$. The effect of anxiety on heart rate variability, 


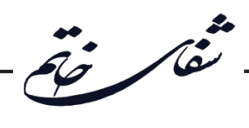

depression, and sleep in chronic obstructive pulmonary disease. J Psychosom Res. 2013; 74(5): 407-13.

21. Loomba RS, Arora R, Shah PH, Chandrasekar S,
Molnar J. Effects of music on systolic blood pressure, diastolic blood pressure, and heart rate: a metaanalysis. Indian Heart J. 2012; 64(3): 309-13. 